\title{
SYMMETRICAL DEGENERATION OF THE NEOSTRIATUM IN CHINESE INFANTS
}

\author{
BY \\ DR. W. J. C. VERHAART \\ Neuropathologist at the School of Medicine, Batavia.
}

In 1934 I described (Verhaart, 1934) three rare cases of cerebral degeneration in Chinese infants, two of them showing extensive symmetrical degeneration in the caudate nucleus and the putamen with formation of large neuroglial nuclei, resembling Alzheimer's cells, while the other case showed the same affection in several parts of the cerebral cortex. For this reason the three cases were compared with the hepato-cerebral degeneration of Kinnier-Wilson and Strümpell-Westphall. It is not certain that these three cases telong to the same type of affection, as there are important differences between them and similar cases in families belonging to the Wilson-pseudosclerosis group, which have not yet been described in Europe. But now I have examined three other cases resembling greatly the second one formerly recorded, and the resemblance is so important that $I$ feel justified in bringing them into one group and in supposing that they have the same etiology.

\section{Case records.}

The second patient in the above-mentioned paper was a Chinese girl, eight months of age, who for thirteen days had suffered from fever, cough and stiff limbs. She had not been ill before and was entirely breast fed. During the three days before death the patient was in coma, there was rigidity of the neck, but the stiffness of the limbs diminished. At the post-mortem examination the liver showed a severe degree of fatty degeneration, the brain was not oedematous, but the nucleus caudatus and the putamen were softened and somewhat gelatinous on the surface of a section.

When examined by microscope the neostriatum showed large foci of spongious structure, proliferation of the capillaries, increased nuclei of the neuroglia and almost total degeneration of the neurons and white matter (fig. 1) ; normal tissue had all but disappeared. In the glotus pallidus neuroglial tissue was somewhat increased in size, but the ganglion cells were 
unaffected. In the medial thalamic nucleus and the superior colliculus the same changes were found to a lesser degree.

I have seen the same histological changes three times in the subsequent years. It is now possible, therefore, to unite these four cases in one group and to compare them with each other.

The second child was a Chinese boy T. T. L., born December 29, 1934, who was admitted in the Central Hospital May 29, 1935, having been ill for nine days. He suffered from fever and kept his eyes constantly closed. There was no cough and no diarrhoea. He had been entirely breast fed. The family consisted of four other children who were healthy. During his stay in the hospital no convulsions were seen, respiration was irregular and the patient

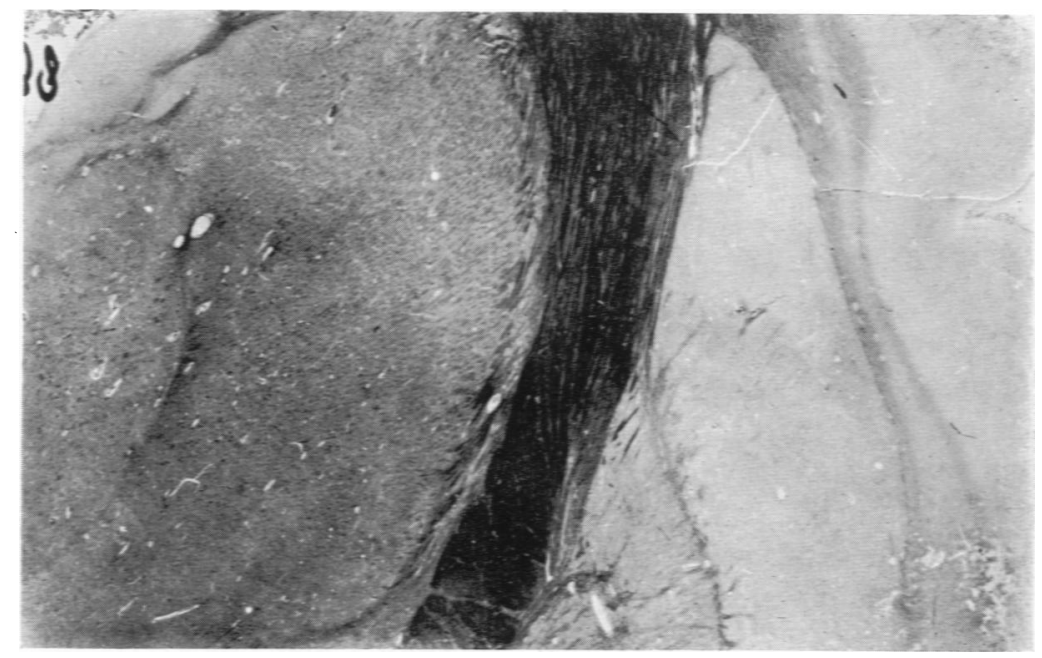

FIG. 1.-Weigert-Pal section of the lenticular nucleus and thalamus of case 1, the myelin sheaths in the putamen have disappeared, but in the globus pallidus and the thalamus they are unaffected.

was drowsy. There were no signs of meningitis. The Wassermann reaction was negative in the patient's blood and the blood of his mother. No changes were found in the cerebrospinal fluid ; lead lines could not be detected in the x-ray pictures of the skeleton. Death occurred on June 2, 1935, three days after the beginning of the disease.

At the post-mortem examination a bilateral bronchopneumonia was found with congestion and fatty degeneration of the liver. The brain was normal externally, but on the cut surface the putamen and the caudate nuclei were softened and somewhat gelatinous. In the putamen and caudate nuclei of both sides extensive foci were found, containing vascular proliferation, distended globular neuroglial nuclei, swollen oligo-dendroglia cells and almost total degeneration of the ganglion cells (figs. 2, 3). In the globus pallidus, the corpus subthalamicus and the superior colliculus a small number of similar foci were present, while in the reticular substance of the midbrain, the pons, the medulla oblongata and the spinal cord a relatively large number of swollen neurogiial nuclei were found. In the central grey matter around the iter of 


\section{DEGENERATION OF NEOSTRIATUM}

Sylvius the ganglion cells were somewhat degenerated; they were swollen, contained a great amount of lipofuchsine and the nucleus in many of them was eccentric. Moreover there was a distinct degree of neuroglial proliferation.

In the cerebral cortex there were a small number of rod cells and other
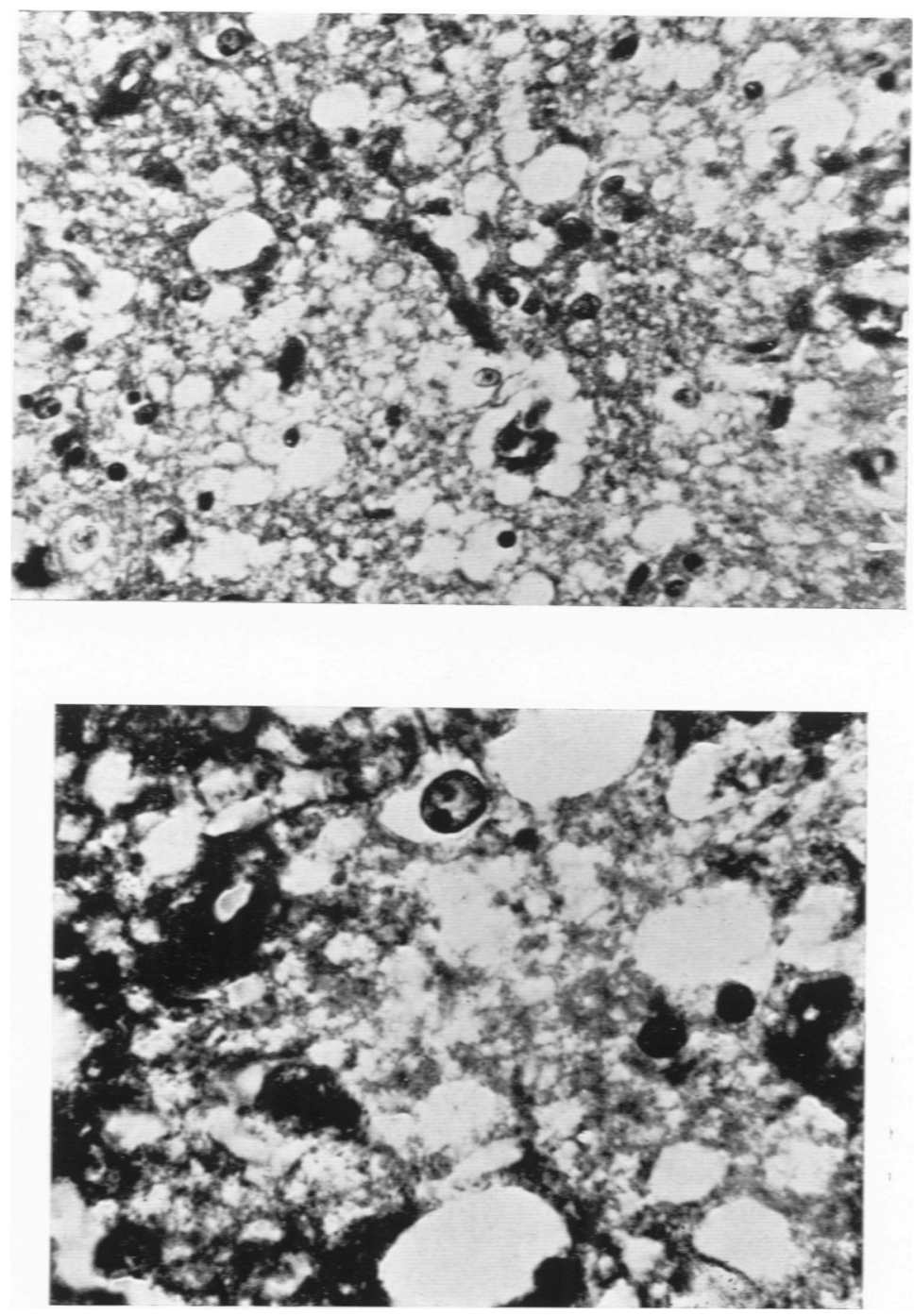

FIGS 2 and 3.-Lower and higher magnification of haematin-eosin sections of the putamen of case 2 : note the spongious structure.

forms of enlarged microglial cells, and the vessel walls were thickened; in the white matter the neuroglial nuclei were enlarged, the vessels were engorged. In this case too there was degeneration of the neostriatum with only small foci in some other parts of the central nervous system, an unspecific reaction in the cerebral cortex and fatty degeneration of the liver. 
The third child suffering from the same disease was the Chinese girl F. O., five months of age, who entered the hospital one hour before death. She had been ill for eight days with fever and coughing. She was the youngest of five children, two of whom had died; she was entirely breast fed. At the hospital the signs of bronchopneumonia were found, the nutritional state was good. At the post-mortem examination slight fatty degeneration of the liver was present, the brain was externally congested, on the cut surface the putamen and the caudate nucleus on both sides were yellowish, gelatinous and softened. In the cerebral cortex and white matter the same unspecific changes were found as in the former case, with some small spongious foci around the vessels in the white matter containing some scavenger cells.

The putamen and the caudate nuclei showed the same changes as in the other cases, but there were a small number of extensive foci, extending to the margins of these grey nuclei. In the centre of these foci the tissue was almost

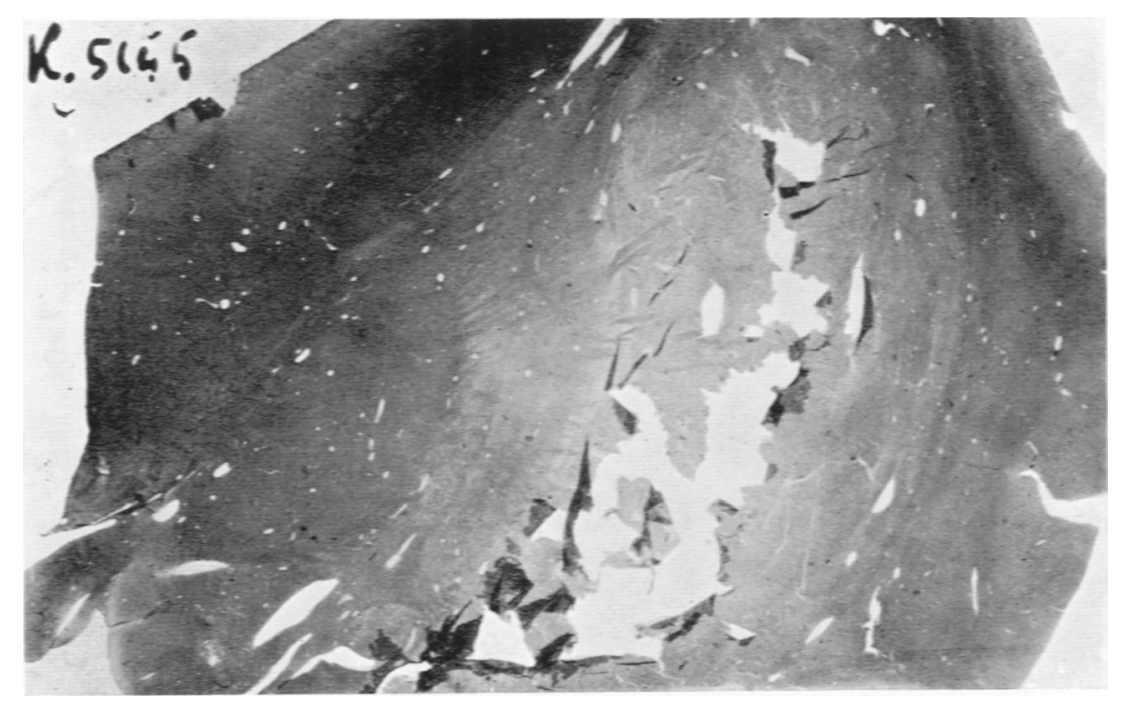

FIG. 4.-Frozen section of the lenticular nucleus of case 4 : the putamen has been torn because of the presence of softening.

'emaciated,' only a network of glial fibres and small vessels with a small number of scavenger cells remaining. The neuroglia cells were enlarged and resembled Alzheimer's glia cells type two. These cells were larger than those of the former patient without reaching the size of the Alzheimer cells found in cases of pseudosclerosis.

In the other parts of the central nervous system no changes were found, but a small amount of lead was found to be present (9.9 $\gamma$ per gramme dry tissue).

The fourth child was a Chinese boy T. W. H., born January 17, 1937, who at the end of July, 1937, grew weak and almost lost his voice. On August 5, he developed fever and the following day he was admitted to hospital. His temperature was then $39 \cdot 3^{\circ} \mathrm{C}$.; he was very drowsy and had many convulsions. The tone of the limbs was diminished, the turgor of the skin was bad, there was no rigidity of the neck, the anterior fontanelle was somewhat sunken, the pupils were of normal size and reacted to light. The cerebro-spinal fluid was 
clear, Nonne and Pandy's reactions were feebly positive, 5 cells per c.mm. were found ; the Wassermann-reaction was negative, but Meynicke II was feebly positive ; micro-organisms were not found.

The patient had not been vaccinated, his parents and relations were healthy, only talcum powder had been used ; in the x-ray pictures no lead lines were present in the bones. The child had been entirely breast fed. On the day after admission the child died.

On naked-eye examination the central nervous system was quite normal, but at the cut surface the caudate nucleus and the putamen were yellowish and softened (fig. 4).

Microscopically the same changes were found as in the preceding cases, viz. spongious structure with enlargement of the neuroglial cells in the caudate nucleus and the putamen with moderate increase and enlargement of the

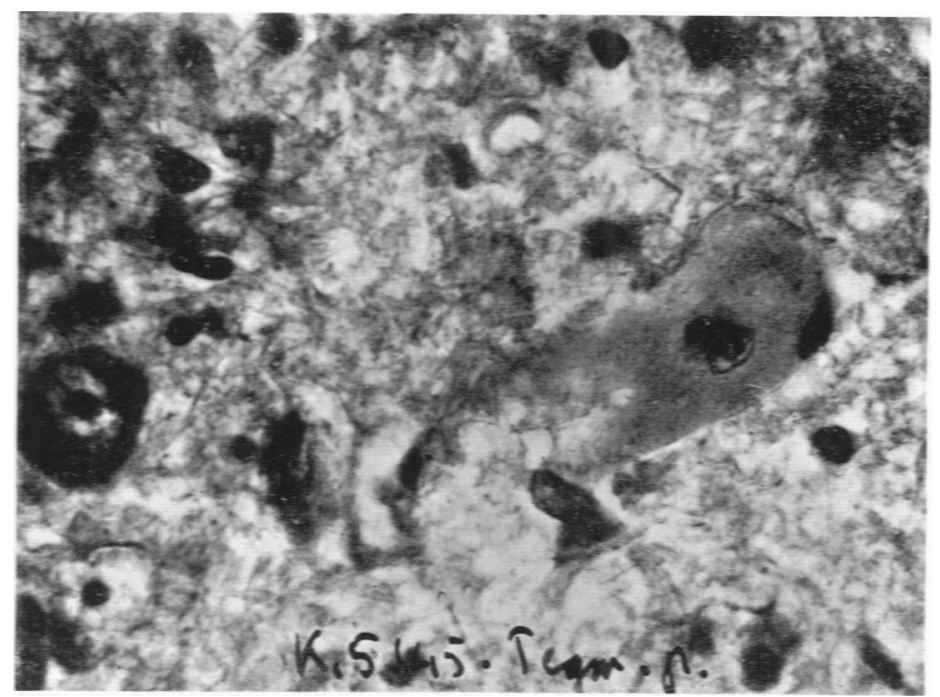

FIG. 5.-Section of the central grey matter in case 4, stained with haematin and eosin ; degenerated and homogenized neuron. The nucleus seen in its centre was found to be situated outside the cell and does not belong to it.

microglia and swelling of the oligo-dendroglia cells. The capillaries were proliferated, some vessels were surrounded by a small number of compound concrements and a small number of vessels by some scavenger cells. The ganglion cells were not in the first place damaged, for nowhere were they totally absent; even in the most affected spots where the tissue was all but ' emaciated' some of them could be seen. However, they were diminished in number in whole of the affected grey nuclei, the small ones had lost most of their protoplasm and the big ones were deformed.

In the frontal half of the striatum the changes were most intensive, but the border of the putamen situated near the marrow of the insula was almost unaffected, while in the caudal half only a small spot at the border of the globus pallidus was degenerated. At that place the globus pallidus showed some proliferation of the neuroglia and some breaking-up of the tissue, but everywhere else it was without lesions.

In the cortex and the white matter of the brain no severe changes were 
found, but spongious structure and proliferation of the neuroglia were also present in the medial half of the corpus subthalamicum, in the central portion of the red nucleus, in the colliculi and the central grey matter around the aqueduct. In this area severe degeneration of ganglion cells could be demonstrated, a number of them showing total homogenization, in this way changing to large homogenous masses that resembled the neurons only in exterior shape (fig. 5). These were the same changes as in the second child, but to a much more severe degree.

Lead proved to be absent in the central nervous system.

\section{Clinical and pathological summary}

Altogether four children have been examined, all of them Chinese infants, eight, five, six and six-and-a-half months of age respectively, who died after suffering for a short period from unspecific cerebral disturbances with coma. Only the first one, the eldest, showed increased tone of the muscles of the limbs ; with the others symptoms of affection of the cerebrum were lacking. All of them were exclusively breast fed, tuberculosis and syphilis could not be found ; lead was demonstrated in a small quantity in the third case, but in the fourth it was absent. Familial or hereditary diseases could not be found; moreover, the second patient was the fifth child out of five, the others being perfectly healthy.

At the post-mortem examination in all of the four cases an extensive symmetrical disintegration of the striatum was found in so typical a manner that in the last case the diagnosis could be made macroscopically. Microscopically a general or localized rarefication of the tissue of the caudate nucleus and the putamen was found with proliferation of the macroglia, which to a greater or lesser degree gave origin to increased naked nuclei, resembling Alzheimer's cells of the second type. The neurons in all cases were scanty, in some of them practically absent ; the capillaries were increased in number, granular cells were present in small quantity, the microglia was increased in size and number, the oligo-dendroglia cells were swollen. To a lesser extent the same changes were found in the globus pallidus, especially at the border of the putamen, the corpus subthalamicus and the red nucleus and the corpora quadrigemina in some cases. In the second and the fourth case ganglion cell degeneration and gliosis were present in the central grey matter, while in the reticular nuclei of the brain stem glial proliferation and a slight degree of rarefication of the tissue existed. In all cases fatty degeneration of the liver proved to be present.

\section{Discussion}

It is obvious that these cases show so much similarity, that they must be looked upon as belonging to the same kind of affection and that it is justifiable to suppose the same etiology. In my former paper, in which the first case has already been mentioned, it was put together with two other cases, that differed much more and they were compared with the diseases of the Wilson-pseudo- 
sclerosis group. Certainly the four cases here described must be compared with this group, although the typical characteristics must be more critically dealt with than with adult cases. Especially the great neuroglial nuclei, resembling Alzheimer's cells, are much more frequently found in children's brains than in those of adults, a fact that Sterren noted in his thesis in connexion with my former paper. In my Batavian material, consisting of about 100 brains of children below the age of two years, they were found with different diseases, e.g. lead encephalopathy, bacillary dysentery and in cases of convulsions of unknown origin. Fatty degeneration of the liver was also frequently found, but not always ; in some cases of severe fatty degeneration the abovementioned neuroglial nuclei were absent. They were found especially in the pieces of grey matter that connect the caudate nucleus with the putamen and in the latero-dorsal margin of the putamen, while in the deep layers of the cortex and the white matter near to it they were also frequently to be found. The large typical Alzheimer cells, however, were rare, most of the nuclei were only somewhat bigger than the normal ones and the chromatine within the nucleus was scanty. They were not present in normal infants in their typical appearance, but everywhere, especially in the white matter of the cerebral hemispheres, the neuroglial nuclei were bigger and more regularly globular than in adults. In the course of the first year they gradually grow smaller, they contain more chromatin and lose their globular shape in the same measure as the fibre-forming spider cells increase in number. But even at the end of the first year the globular nuclei are still relatively numerous. Therefore it is obvious that with infants the large naked globular nuclei come into existence more easily than in adults, in whom the neuroglia has a tendency to form fibres and a glious scar.

Notwithstanding these facts a comparison of my four cases with Wilson's disease is justifiable because of the extensive symmetrical disintegration of tissue in the neostriatum. Identification will be difficult, since no heredity could be proved, no cirrhosis of the liver occurred and in European families suffering from hepatocerebral degeneration such cases have not been described in infancy. With my cases it is even uncertain that a connexion can be accepted between the degeneration of the liver and the striatum because fatty degeneration so frequently occurs with infants.

In Western literature only a few similar cases could be found, First there is a case by Marinesco and Draganesco (1929) published under the title "A case of Förster's Syndrome.' A four-year-old child after an infectious disease showed diplegia with athetosis, anarthria and idiocy. When the child was lying, the legs were flaccid, but when the child was trying to stand they were stiffly stretched; half a year after the beginning of the disease the child developed bedsores and died. At the post-mortem examination fatty degeneration was found in the heart muscle and in the liver ; the putamen and the caudate nucleus were symmetrically brown and atrophic ; in both centra there was disintegration with a slight grade of 'état lacunaire' and degeneration of the white matter in the globus pallidus at the margin of the putamen. The neurones in the striatum had almost disappeared; in the frontal part there was a great number of granular cells around the vessels, the micro- and 
macroglia had proliferated, glial fibres were formed, the vessels were increased in number, their walls were thickened. In the cerebral cortex the ganglion cells were scanty ; the Purkinje-cells in the cerebellum had also diminished in number.

It is probable that the patients described here would have shown almost the same changes had the disease begun at a somewhat higher age and had the time between the beginning and death been longer. The resemblance is definitely present : the writers cannot find any etiological factor ; they do not think the identity with Wilson's disease absolute, especially because of the affection of the liver.

Icterus gravis neonatorum with 'kernicterus' of the German writers must be mentioned, but the differences are greater than the similarities. There was no severe icterus shortly after birth and the central grey matter was not stained intensively yellow.

Some similarity is found in the cases described by Paterson and Carmichael (1924) as a familiar degeneration of the lenticular nucleus with a family of twelve children of which only two were healthy. The others died before they were ten months of age with the exception of one, who lived up to two years of age. They all showed cerebral symptoms, the twelfth child was born normally, but soon it grew cyanotic and died three days later. The lenticular nucleus showed proliferation of the vessels and the glia, and small necrotic foci with granular cells occurred. Large irregularly formed glial cells were present, the ganglion cells in the putamen and the globus pallidus had all but disappeared ; in the caudate nucleus two small necrotic spots with granular cells occurred. In the cerebrum and the thalamus unspecific changes were found.

The eleventh child, who died at the age of seven months, showed decreased muscular tone and power, with impaired vision for some months. After death the same changes in the lenticular nucleus occurred, but in the cerebral cortex, especially in the occipital regions, many ganglion cells were missing, while the white matter could only be stained with difficulty. In the substantia nigra, also, the glia and the vessels were proliferated, but the ganglion cells had only disappeared to a lesser degree. The authors are of opinion, that a toxin, circulating in the blood, can be the cause of the degenerations, the peculiar course of the vessels within the lenticular nuclei being the reason of the severe degeneration at that place.

As is obvious, the points of similarity are less than with the patient described by Marinesco and Draganesco (1929), especially concerning the changes in the substantia nigra and the cerebral cortex as well as the familial incidence.

As starting-points for a discussion of the etiology are the facts that the disease began in all of the cases at about six months of age in Chinese children exclusively fed on the breast. At that age breast feeding usually begins in Batavia to become insufficient, as de Haas demonstrated some time ago, the body weight increasing less than in European infants about that time. Naturally this cannot be the only factor, because this method of feeding is common in the Indies, but perhaps it can facilitate it when other toxic instances occur. Possible poisoning of the children by the mode of living or dressing of the 
Chinese must also be taken into account ; de Langen proved that the degeneration of the white matter in the cerebrum only occurring in Chinese children was caused by lead intoxication. Originally an endogenous racial factor was supposed to exist, but it proved to be the habit with Chinese inhabitants to use lead-containing toilet powder because of its greater covering power, while the natives use rice and the Europeans talcum powder.

In the present cases this cannot be the reason, because lead certainly was absent in one of them and the typical lead lines in the bones were absent in two cases, but manganese must still be taken in account. With adults it is known that manganese can bring about degeneration of the striatum, causing tremors and increased tone of the muscles.

Caravan, Cobb and Drinker (1934) describe such a case in a man, who at the age of fifty-four developed similar neurologic disturbances that diminished as soon as he stopped working with manganese. After his death at sixty-eight atrophy of the lenticular nucleus was found with degeneration of the neurons, but without disintegration of the tissue or proliferation of the neuroglia. Therefore the resemblance is not great and the probability that manganese has something to do with the cases here described is slight.

Endogenous racial factors can be considered for the differences between disease in the Indies and Europe probably do not wholly depend on exogenous causes. It is certain that multiple sclerosis and Huntington's chorea are much less frequent here than in Europe or America ; with Japanese this type of chorea has not yet been found and in the Indies only two cases have been described. With natives the great frequency of primary cancer and cirrhosis of the liver and the rarity of cancer of the stomach are remarkable facts that have not yet been altogether explained. Notwithstanding the frequency of cirrhosis of the liver, pseudosclerosis has not yet come to autopsy in natives although mild specific changes of the neuroglia in the striatum have been found frequently in cases of cirrhosis of the liver. Once only a patient suffering from the cirrhosis showed tremors and increased tone of the limbs for some weeks before death ; in the striatum neuroglial changes and mild degeneration of ganglion cells were present.

To sum up : at the moment it is impossible to express an opinion about the differences in pathology between races, but these cases are described as interesting contribution to the causation of degeneration of the striatum.

Postscript : I have only now become acquainted with the paper of A. Biemond and S. v. Creveld (1937), on nuclear jaundice in neonatal sepsis with jaundice. The post-mortem findings have something in common with those in the present cases, namely degeneration of the corpus subthalamicum and the pallidum, but the putamen is unaffected. Moreover, the clinical history showed great differences : in their cases the infants were seriously ill shortly after birth with high fever and jaundice, they developed their neurological symptoms at that time and these symptoms remained unchanged until death occurred several months later. I therefore think that the cases here described cannot be of the same origin. 


\section{REFERENCES}

Biemond, A., and v. Creveld, S. (1937). Arch. Dis. Childh., 12, 173.

Caravan, M. M., Cobb, S., and Drinker, C. K. (1934). Arch. Neurol. Psychiat., Chicago, $32,3$.

de Langen, C. B. (1934). Geneesk. Tijdschr. v. Ned-Indie, 74, 3.

Marinesco, G., and Draganesco, S. (1929). Encephale, 24, 8.

Paterson, D., and Carmichael, A. (1924). Brain, 47, 207.

Verhaart, W. J. C. (1933). Z. ges. Neurol. Psychiat., 147, 1.

(1934). Ibid., 150, 4.

(1937). Acta Paediat., 30, 107. 